\title{
REVIEW
}

\section{Quantitative EEG for the detection of brain ischemia}

\author{
Brandon Foreman' and Jan Claassen ${ }^{2 *}$ \\ This article is one of eleven reviews selected from the Annual Update in Intensive Care and Emergency Medicine 2012 (Springer Verlag, \\ DOI: 10.1007/978-3-642-25716-2) and co-published as a series in Critical Care. Other articles in the series can be found online at \\ http://ccforum.com/series/annualupdate2012. Further information about the Annual Update in Intensive Care and Emergency Medicine is \\ available from http://www.springer.com/series/8901.
}

\section{Introduction}

Monitoring in most intensive care units (ICUs) is limited to continuous assessments of cardiopulmonary function, whereas brain monitoring has traditionally been limited to serial neurological examinations and infrequent imaging studies. Increasingly it is becoming clear that secondary neurological complications, such as seizures and brain ischemia, are also seen in the medical-surgical ICU population and are not limited to patients with primarily neurological injury. Electroencephalography (EEG) offers a continuous, real-time, non-invasive measure of brain function. Originally developed for the characterization of seizures and epilepsy, continuous EEG monitoring (cEEG) has been used for seizure detection in the ICU. Additionally, cEEG has been used as a method of identifying subclinical brain injury during neurosurgical procedures, such as carotid endarterectomy, and for ischemia detection, global function assessment, medication titration, and prognostication [1].

In the ICU, ischemia detection in particular has been under-utilized, but the potential to diagnose ischemia as it occurs is tremendous (e.g., monitoring after high risk vascular or cardiac surgery, during refractory hypotension, or in the context of sepsis-associated encephalopathy). When cerebral blood flow (CBF) becomes compromised, changes occur in both the metabolic and electrical activity of cortical neurons, with associated EEG changes [2]. In the operating room, EEG has an established role in identifying ischemia prior to the development of infarction during carotid endarterectomy [3]. In acute ischemic stroke, the primary injury has typically occurred prior to presentation, but EEG may be

\footnotetext{
*Correspondence: jc1439@columbia.edu

${ }^{2}$ Department of Neurology, Columbia University, New York, NY, USA

Full list of author information is available at the end of the article
}

able to detect patterns to suggest severity, prognosis, and secondary injury (e.g., reocclusion, edema, or hemorrhagic transformation) [4]. Delayed cerebral ischemia from vasospasm after subarachnoid hemorrhage (SAH) illustrates an application in which early detection may prevent the development of permanent damage by triggering appropriate interventions such as angioplasty or intra-arterial administration of vasodilator therapy $[5,6]$. Serial neurological exams and imaging are only capable of detecting delayed cerebral ischemia once the damage becomes clinically or radiographically apparent. In this case, EEG may be a useful way to detect and subsequently treat ischemia before the injury becomes irreversible [7-10].

\section{EEG changes in ischemia}

Brain function is represented on EEG by oscillations of certain frequencies. Slower frequencies (typically delta $[0.5-3 \mathrm{~Hz}]$ or theta $[4-7 \mathrm{~Hz}])$ are generated by the thalamus and by cells in layers II-VI of the cortex. Faster frequencies (or alpha, typically $8-12 \mathrm{~Hz}$ ) derive from cells in layers IV and V of the cortex [11]. All frequencies are modulated by the reticular activating system, which corresponds to the observation of reactivity on the EEG [12]. Pyramidal neurons found in layers III, V, and VI are exquisitely sensitive to conditions of low oxygen, such as ischemia, thus leading to many of the abnormal changes in the patterns seen on EEG [4].

EEG changes are closely tied to CBF (Figure 1) [3]. When normal CBF declines to approximately 25$35 \mathrm{ml} / 100 \mathrm{~g} / \mathrm{min}$, the EEG first loses faster frequencies, then as the CBF decreases to approximately 17$18 \mathrm{ml} / 100 \mathrm{~g} / \mathrm{min}$, slower frequencies gradually increase. This represents a crucial ischemic threshold at which neurons begin to lose their transmembrane gradients, leading to cell death (infarction). In the setting of carotid 


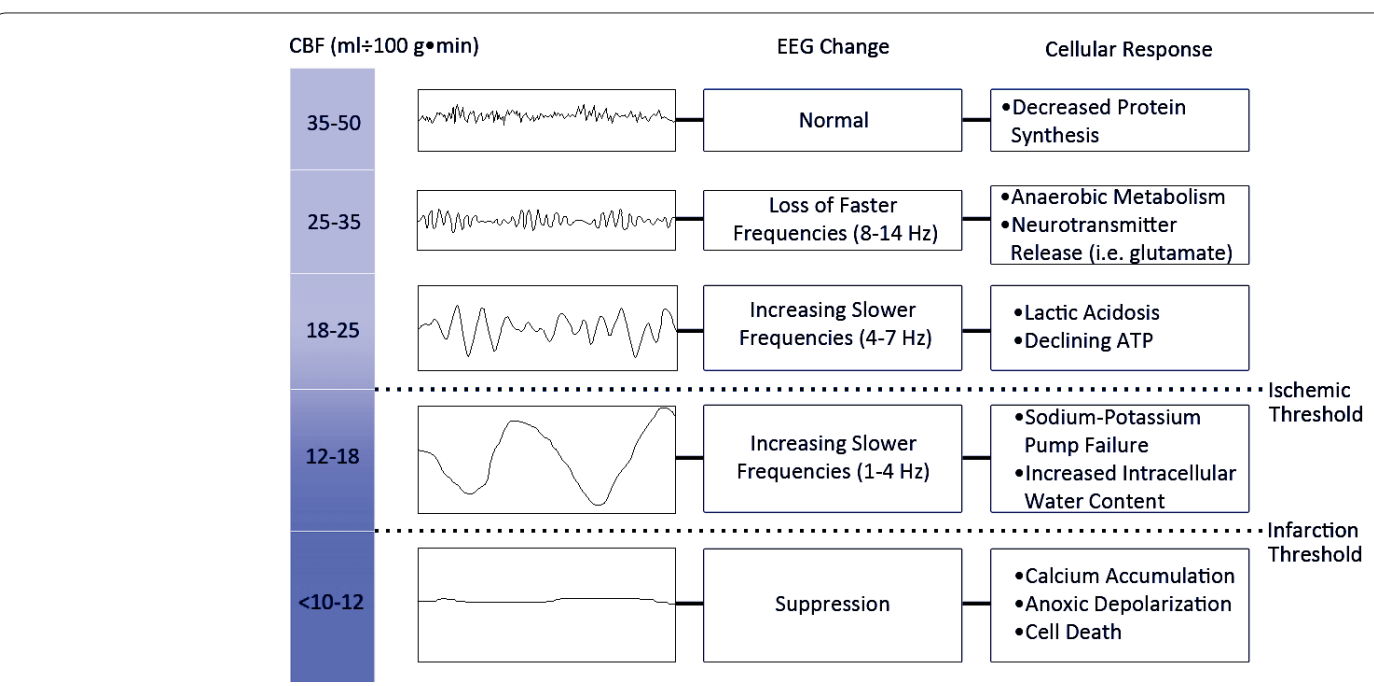

Figure 1. The relationship of cerebral blood flow to electroencephalogram (EEG) and pathophysiology. ATP, adenosine triphosphate (CBF). Data from $[2,4]$.

clamping, CBF that decreases instantaneously to the ischemic threshold leads to rapid and reversible changes in the EEG (within 20 seconds) [3]. Infarction may not occur for hours at this degree of flow limitation [2] and some electrical activity (mostly delta frequencies) may be seen, but as the CBF continues to decrease toward the infarction threshold (10-12 $\mathrm{ml} / 100 \mathrm{~g} / \mathrm{min}$ and below), the EEG becomes silent and cellular damage becomes irreversible [2-4]. Some EEG patterns, such as regional attenuation of faster frequencies without delta (RAWOD), may reflect early severe loss of CBF (mean $8.6 \mathrm{ml} / 100 \mathrm{~g} /$ min) as seen during large occlusive infarcts, leading to complications such as edema and herniation [13].

Studies of CBF and cerebral rate of oxygen metabolism $\left(\mathrm{CMRO}_{2}\right)$ using Xenon computed tomography $(\mathrm{CT})$ and positron emission tomography (PET) in ischemic stroke have demonstrated that regional EEG changes also reflect the coupling of CBF and metabolism [14]. In early subacute ischemic stroke, the EEG correlates well with CBF as the oxygen extraction fraction increases to preserve $\mathrm{CMRO}_{2}$, a period termed "misery perfusion" or stage 2 hemodynamic failure [14,15]. Later, the EEG appears to correlate less well with $\mathrm{CBF}$ and instead begins to correlate with $\mathrm{CMRO}_{2}$ during a period of "luxury perfusion" or stage 3 hemodynamic failure $[14,15]$. Early in this dynamic relationship, the threshold for cellular damage is shifted such that neuronal loss and decreased protein metabolism may in fact precede changes related to critical CBF, particularly in peri-infarct areas [2]. These areas are also complicated by abnormal glutamate release (between 20-30 ml/100 g/min) or peri-infarct depolarizations $[2,16]$.

The transition from ischemia to infarct occurs over a range of $\mathrm{CBF}$, potentially providing a window of opportunity to treat and reverse impending neuronal cell death.
Diffusion-weighted magnetic resonance images (DWI MRI) are capable of detecting changes at CBF 35$40 \mathrm{ml} / 100 \mathrm{~g} / \mathrm{min}$ within 30 minutes [17]. In contrast, EEG detects changes at the same CBF within seconds and allows for continuous monitoring of these changes over time. This can be crucial to detect evolving ischemic changes after treatment with tissue plasminogen activator ( $\mathrm{tPA}$ ), when the $\mathrm{CT}$ is negative during early infarction, or when there is a mismatch between DWI MRI and the clinical examination. Additionally, EEG may assist in guiding treatment, to rapidly reverse the region at risk if it has not reached the infarction threshold. Examples include shunt placement in carotid endarterectomy [3], tPA in acute ischemic stroke $[18,19]$, and augmentation of blood pressure or CBF after acute ischemic stroke [20].

There are some data suggesting that EEG may help with prognostication in patients with acute ischemic stroke. Because cortical changes are modulated by brainstem reticular formations, global changes, such as loss of reactivity [21] and lack of sleep-wake cycles [22], may portend poor prognosis (and indicate possible brainstem involvement). Regional changes, including a lack of delta or the presence of faster frequencies within 24 hours, correlate with a good outcome [23] while the persistence of unilateral prominent, continuous delta slowing or decreased alpha adds significantly to clinical predictions of poor outcome [24]. Other specific patterns may also indicate poor prognosis, such as RAWOD (with up to $67 \%$ mortality) [13] or periodic lateralized epileptiform discharges (PLEDs) [25].

\section{A need for quantitative EEG}

Despite the potential to detect ischemia, there are a number of limitations that need to be overcome before 
using this technique for automated event detection in ICU practice. Early obstacles, such as data acquisition, remote access, and data storage have been largely overcome by technological advances. Nonetheless, raw EEG requires interpretation by trained experts, ideally with experience in continuous monitoring for critically ill patients. Subtle changes over time (hours to days) may be missed and visual analysis can be extraordinarily timeconsuming. To achieve the goal of real-time detection of critical brain events, raw EEG would require continuous expert review. Although telemedicine has become a prominent tool in acute stroke, centralized EEG monitoring with access to around-the-clock neurophysiology experts is not currently available and would be extremely expensive.

The most pressing limitation to cEEG remains its interpretation, which is ultimately subjective. By applying a Fourier transformation, EEG can be quantified in terms of its amplitude, power, frequency, and rhythmicity in order to generate numerical values, ratios, or percentages; graphically display arrays or trends; and set thresholds for alarms. A variety of quantitative EEG (qEEG) measures have been used clinically to quantify slowing or attenuation of faster frequencies in the EEG, specifically: The calculation of power within different frequency bands (i.e., delta, theta, alpha, and beta); ratios or percentages of power in specific frequency bands; and spectral edge frequencies (based on the frequency under which $\mathrm{x} \%$ of the EEG resides). These discrete values can then be compared between different regions (between hemispheres, for instance, or between electrode-pair channels). Time-compressed spectral arrays were developed to incorporate both power and frequency spectrum data and are reconstructed using color to represent power at different frequencies (a so-called 'spectrogram'). These spectrograms have the highest pixel-to-data ratio, yielding easy-to-digest color representations of large amounts of data within a single screen (compared with the raw EEG) [26]. Additional measures include amplitude integrated EEG, which was developed to continuously monitor comatose patients via average ranges of peak-topeak amplitudes displayed using a logarithmic scale, and the commercial Bispectral Index, often used to monitor the depth of anesthesia (Aspect Medical Systems, Inc., Newton, MA). Other nonparametric methods exist beyond Fourier transformation, including interval or period analysis and alternative transformation techniques. Parametric, mimetic, and spatiotemporal analyses are also available using a variety of computational methods that are beyond the scope of this review [27]. For now, fast Fourier transformation appears to be the most suitable for quantifying the EEG [28] but future algorithms may also incorporate ICU-specific EEG feature recognition and waveform analysis based on machine learning approaches trained on ICU EEG recordings.
It is important to emphasize that there are a large number of confounders to be considered when evaluating EEG signals. Both raw EEG and qEEG can be altered in response to the presence of seizures or periodic discharges, changes in intracranial pressure (ICP, e.g., acute hydrocephalus), or in the setting of systemic illness such as ventilator-acquired pneumonia (VAP). In addition, sedatives, narcotics, antipsychotics, and other medications may have varying effects on the EEG. Even normal state changes, such as slow wave sleep, appear similar to changes seen during ischemia. Moreover, the ICU is contaminated with a variety of artifacts: Chest percussion, bed movement, condensation in ventilator tubing, and electrical $(50 \mathrm{~Hz}$ or $60 \mathrm{~Hz})$ artifact from other monitoring devices. Ultimately, the raw EEG remains the gold standard for the assessment of brain function. Any algorithm based on qEEG should include raw EEG for review by staff familiar with EEG - either on site or remotely $[9,26]$.

\section{Quantitative EEG in ischemia}

Like raw EEG, qEEG is capable of reflecting changes in blood flow and metabolism in as little as 28-104 seconds [29]. However, different quantitative parameters may have their own strengths for a given clinical situation. Intraoperative spectral edge frequencies, for example, detect ischemic changes in carotid endarterectomy patients receiving isoflurane anesthesia, whereas changes in the relative delta percentage (that is, delta power/total power) are accurate in patients receiving propofol [29]. In acute ischemic stroke, hemispheric relative delta percentage, spectral edge frequencies $25 \%$ and $75 \%$, and the overall mean frequency correlated well with CBF [28]. Cerebral perfusion pressure (CPP), related to $\mathrm{CBF}$ and to $\mathrm{ICP}$, correlates with decreases in mean frequency as CPP falls below $60 \mathrm{mmHg}$, even in comatose or sedated patients [30]. Finally, increased power in slower frequency bands (delta and theta) and decreased power in faster frequency bands (alpha and beta) are seen with reductions in brain metabolism $\left(\mathrm{CMRO}_{2}\right)$ [14]. Overall, the relative delta percentage appears to provide the most robust correlation with $\mathrm{CBF}$ and metabolism during focal ischemia.

Clinically, qEEG correlates with stroke severity, radiographic findings, and response to treatment. Several parameters correlate with initial stroke severity as measured by the National Institutes of Health Stroke Scale (NIHSS) in both the acute (brain symmetry index [BSI]) $[19,31]$ and subacute periods (relative alpha percentage, relative alpha-beta percentage, relative delta-theta percentage, delta/alpha ratio, delta-theta/alpha-beta ratio, and global pairwise derived BSI [pdBSI]) [32-34]. qEEG parameters correlate with the volume of infarction on MRI, such as the acute delta change index [35], global pdBSI [32], relative alpha percentage, relative alpha-beta percentage, 
relative delta-theta percentage, delta/alpha ratio, and delta-theta/alpha-beta ratio [36]. In the case of subcortical infarcts, both the delta-theta/alpha-beta ratio and the pdBSI correlate surprisingly well with infarct volumes, possibly related to the consequences of diaschisis on CBF in lacunar brainstem infarcts $[33,36]$. qEEG may be more sensitive than raw EEG to subtle changes $[37,38]$, and some parameters may even detect improvement prior to improvement in the clinical exam. For example, after tPA, a decrease in delta power was documented 100 minutes prior to the start of functional improvement [18] and in a series of 16 patients with moderate stroke, one patient had an improvement in BSI a few minutes prior to clinical improvement after the administration of tPA [19].

qEEG may also provide a method of quantifying shortterm and long-term prognosis. At one year, functional outcome after stroke in one series was predicted correctly by clinical criteria $60 \%$ of the time. The addition of EEG data improved the predictive value to $85 \%$ (for both raw and quantitative EEG) [24]. The acute delta change index correlates with 30-day NIHSS as accurately as initial mean transit time on MRI and actually better than initial DWI volume (emphasizing the link between qEEG parameters and blood flow) [35]; other parameters such as the delta/alpha ratio and the relative alpha percentage also correlate with the 30-day NIHSS [34]. As early as 6 hours after stroke, the pdBSI correlates with the 1-week NIHSS. Subacute abnormalities in pdBSI and, particularly, the delta-theta/alpha-beta ratio are associated with the 6-month modified Rankin score [39]. For deeper infarcts, pdBSI and the delta-theta/alpha-beta ratio correspond to 1-week outcome [33]. Extremely elevated pdBSI may even predict early neurodeterioration from other complications at 1 week [36].

The ability to infer CBF patterns, quantify the severity of brain ischemia, and objectively monitor the response to treatment may be useful across critically ill patient populations. Ischemia detection could alert intensivists to acute ischemia after cardiac valve replacement, for example, and could prompt intra-arterial thrombectomy and prevention of stroke. Titration of vasopressors may be influenced by the detection of ischemic changes despite an otherwise appropriate mean arterial pressure (MAP) goal. Although recent studies of EEG in patients with sepsis have focused on seizure detection and other abnormal epileptiform patterns [40], quantification of cerebral perfusion in these patients could be an invaluable measure of end-organ perfusion to guide pressor management, particularly in patients with sepsis-associated encephalopathy, which may be related to impaired perfusion of the small vessels [41]. SAH provides a useful example to illustrate the need for and potential of existing data related to qEEG techniques in the ICU.

\section{Clinical scenario: delayed cerebral ischemia after subarachnoid hemorrhage}

SAH has an incidence of $6-15 / 100,000$ and is associated with high morbidity and mortality [42]. Much of the outcome is determined by secondary complications many of which are treatable if diagnosed in a timely fashion. Between $20-40 \%$ of SAH patients either clinically deteriorate or have evidence of new ischemia on repeat brain imaging from vasospasm, together termed delayed cerebral ischemia $[5,42,43]$. Delayed cerebral ischemia is associated with worse outcome [5], but may be particularly challenging to diagnose in the comatose patient [6].

The effects of vasospasm-mediated delayed cerebral ischemia can be mitigated with pressure and volume augmentation (so-called triple- $\mathrm{H}$ therapy), intra-arterial vasodilators, such as verapamil, or angioplasty. The benefit of these measures relies on the earliest possible detection before neuronal damage becomes irreversible. Serial neurological exams and radiological confirmation of ischemia offer post hoc diagnosis, but do not adequately detect preclinical events. Currently, the only serial detection method in routine use is transcranial Doppler ultrasonography (TCD). Although mean blood flow velocities of $>120 \mathrm{~cm} / \mathrm{s}$ are around $60-70 \%$ sensitive to changes in the proximal middle cerebral artery (MCA), this cut-off is less reliable (and less well studied) for other territories and does not include the distal vasculature [42]. When used daily, TCDs have been $60-70 \%$ sensitive for delayed cerebral ischemia with limited predictive value; they also exhibit frequent false negatives or false positives [44].

\section{EEG changes in subarachnoid hemorrhage}

SAH produces a wide variety of abnormalities on EEG. Even before the onset of vasospasm, EEG may detect disorganization or slowing, disruption of the normal posterior dominant rhythm, seizures (6\%), periodic discharges $(16 \%)$, a lack of reactivity (14\%), or a lack of sleep transients (in up to 85\%) [45,46]. In a serial EEG study of 151 patients with $\mathrm{SAH}$, the authors found that even on day 1 , certain patterns predicted later vasospasm, including the presence of very brief frontally predominant biphasic delta waves ('axial bursts'), focal polymorphic delta overlying an area of clot, or a predominance of unreactive delta frequency slowing. By day 5, the development of continuous polymorphic, rhythmic, or unreactive delta predicted vasospasm $100 \%$ of the time. These patterns correlated well with CT grades of SAH severity and $40 \%$ of patients with abnormal EEG on day 5 had neurodeterioration [47]. As predicted by the EEG's close correlation with CBF and metabolism, raw EEG was capable of detecting developing ischemia up to $78 \%$ of the time in one study of poor-grade SAH patients [9].

The use of qEEG for ischemia detection in SAH has been examined systematically in a total of 89 patients 
Table 1. Clinical summary of quantitative EEG in subarachnoid hemorrhage

\begin{tabular}{|c|c|c|c|c|c|}
\hline First author [ref] & $\mathrm{N}$ & SAH Grade & Clinical Criteria & Outcome & qEEG Results \\
\hline \multirow[t]{3}{*}{ Labar [7] } & \multirow[t]{3}{*}{11} & $\mathrm{HHI}: 2$ & 1) Focal neurological deficit & \multirow{3}{*}{$\begin{array}{l}\text { Ischemic events } \\
(n=18)\end{array}$} & 5 silent infarcts detected by qEEG alone \\
\hline & & HH III: 8 & 2) Global cortical dysfunction & & 4 qEEG changes prior to clinical changes \\
\hline & & HH IV: 1 & 3) Encephalopathy & & \\
\hline \multirow[t]{3}{*}{ Vespa [8] } & \multirow[t]{3}{*}{32} & Awake & 1) Angiographic vasospasm & \multirow{3}{*}{$\begin{array}{l}\text { Vasospasm } \\
(n=19)\end{array}$} & All vasospasm with qEEG changes. \\
\hline & & \multirow[t]{2}{*}{$(H H I-I I I)$} & 2) $T C D$ vasospasm & & \multirow{2}{*}{$\begin{array}{l}\text { 10/19 qEEG changed mean } 2.9 \text { days prior to } \\
\text { vasospasm confirmation. }\end{array}$} \\
\hline & & & $\begin{array}{l}\text { (> } 120 \mathrm{~cm} / \mathrm{s} \text { or Lindegaard } \\
\text { ratio }>3 \text { ) }\end{array}$ & & \\
\hline \multirow[t]{3}{*}{ Claassen [9] } & \multirow[t]{3}{*}{34} & Comatose & $\mathrm{DCl}$ & \multirow[t]{3}{*}{$\mathrm{DCl}(n=9)$} & \multirow{3}{*}{$\begin{array}{l}\text { Raw EEG changed in } 78 \% \text {; } q \text { EEG sensitive to a } 10 \% \\
\text { change in } 6 \text { post-stimulation minutes or } 50 \% \text { change } \\
\text { in only } 1 \text { post-stimulation minute. }\end{array}$} \\
\hline & & $(H H I V-V)$ & 1) Clinical deterioration & & \\
\hline & & & 2) New infarct on $C T$ & & \\
\hline \multirow[t]{6}{*}{ Rathakrishnan [10] } & \multirow[t]{6}{*}{12} & $\mathrm{mF} 3-4$ & $\mathrm{DCl}$ & \multirow[t]{6}{*}{$\mathrm{DCl}(\mathrm{n}=8)$} & qEEG sensitivity with clinical data is $67 \%$. \\
\hline & & $\mathrm{HHI:} 1$ & 1) Clinical deterioration & & \multirow{5}{*}{$\begin{array}{l}3 / 8 \text { qEEG changed more than } 24 \text { hours prior to clinical } \\
\text { change. }\end{array}$} \\
\hline & & $\mathrm{HH} \| \mathrm{l}: 5$ & 2) New infarct on $C T$ & & \\
\hline & & HH III: 2 & & & \\
\hline & & $\mathrm{HH} \mathrm{IV:3}$ & & & \\
\hline & & $H H V: 1$ & & & \\
\hline
\end{tabular}

DCl, delayed cerebral ischemia; HH, Hunt-Hess Grade; mF, Modified Fisher Grade; qEEG, quantitative EEG; SAH, subarachnoid hemorrhage; TCD, transcranial Doppler ultrasonography.

over 4 studies since 1991 (Table 1). In the first of these studies, EEG was found to detect ischemia earlier and better than clinical exam alone [7] in 11 patients, most of whom were Hunt-Hess grade 3. Total power, the alpha/ delta-theta ratio, and the relative delta percentage were performed in only six patients, but were in aggregate $100 \%$ sensitive to detect infarcts if the changes were sustained over a 24-hour period. Total power specifically detected $100 \%$ of the silent infarcts seen on CT; compressed spectral array on the other hand, was $67 \%$ sensitive for ischemia. Four ischemic events had qEEG changes prior to the development of clinical deficits and five ischemic events had changes in total power that predicted silent infarcts when the clinical exam did not. There were only two false positives reported during 62 days of monitoring.

qEEG and xenon CT measures of CBF were used simultaneously in 32 awake SAH patients (Hunt-Hess 1-3) to detect vasospasm, which was confirmed by angiography or TCD [8]. The relative alpha percentage, defined uniquely as $6-14 \mathrm{~Hz}$ frequency power divided by the total power $(1-20 \mathrm{~Hz})$, was calculated over 2 minute windows to generate a histogram periodically throughout the day. The variability of this histogram was then qualitatively scored from 1 to 4 . Decreased variability trended toward decreased CBF. Overall, 19 of 32 patients developed vasospasm, all of whom had decreased variability of the relative alpha percentage. Seven patients without vasospasm had decreased variability, largely due to stroke or increased ICP. Changes in variability occurred in 4 or more of the 6 electrode channels monitored, suggesting this measure reflects a more global change. Importantly, decreased variability occurred a mean of 2.9 days prior to the development of TCD or angiographic vasospasm.

Using a standard electrode array divided into vascular distributions (anterior circulation and posterior circulation), 34 comatose SAH patients (Hunt-Hess 4-5) were evaluated to detect delayed cerebral ischemia; angiography and TCD data were not required to confirm the clinical or radiological diagnosis [9]. Rather than utilizing continuously generated data, 10 sixty-second clips were obtained both at baseline and between days 4 and 6 . The strength of this particular analysis is that each clip was obtained after stimulation - thereby eliminating the variability associated with state changes or sleep. Total power and power in each frequency band were measured in addition to relative alpha, relative delta, the alpha/delta ratio, and the alpha-beta/delta ratio. The coherence of each hemisphere was calculated for both alpha and delta frequencies and a total average frequency was also determined. Different cut-offs were used to determine whether a change was significant: 10,20 , or $50 \%$ changes from baseline. In predicting the 9 of 34 patients with delayed cerebral ischemia, the post-stimulation alpha/ delta ratio correlated best when anterior and posterior regions were considered together, followed by relative delta, alpha-beta/delta ratio, and relative alpha (Figure 2). 


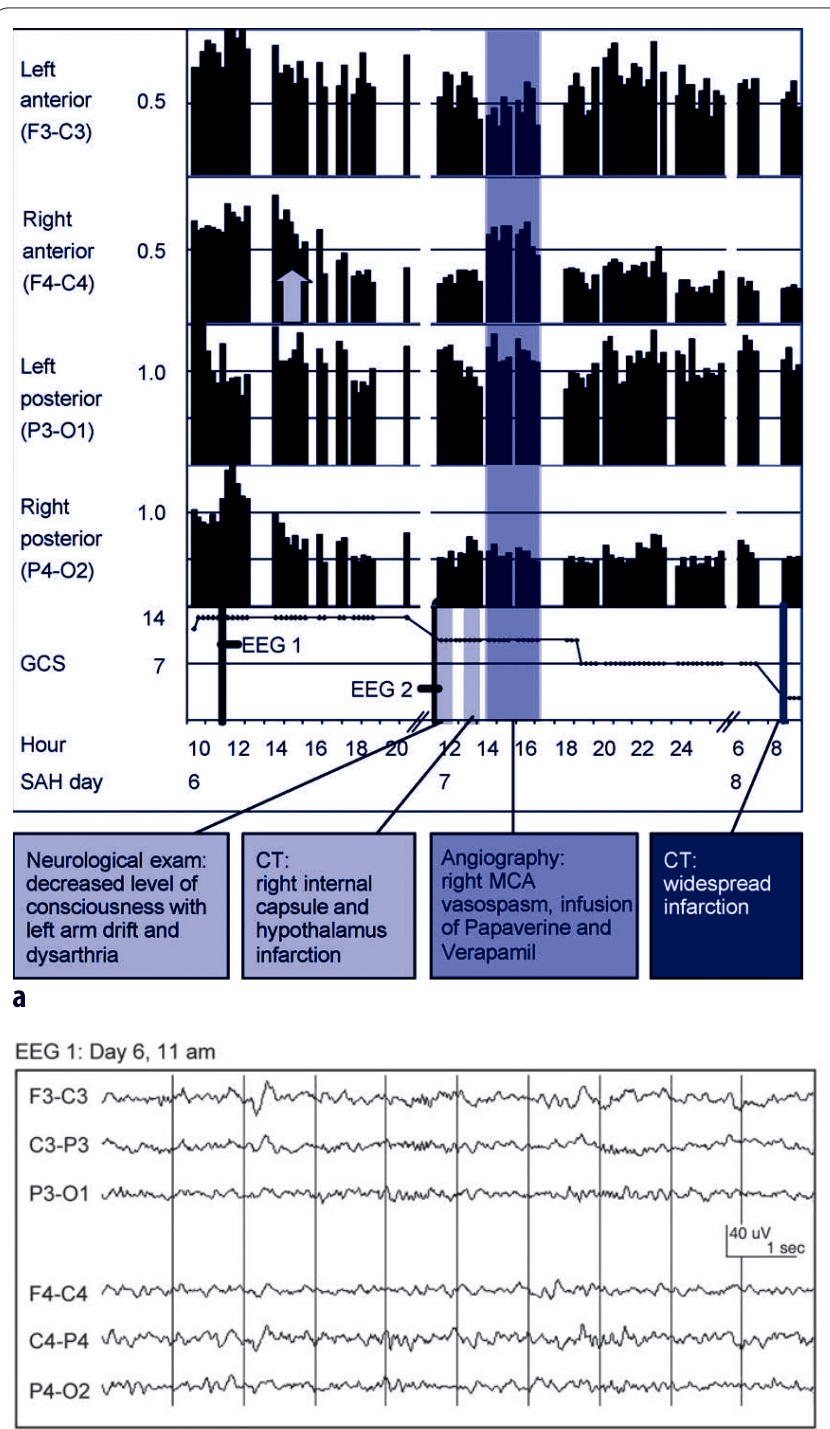

c

Figure 2. (a) Alpha/delta ratio (ADR) calculated every 15 min and Glasgow Coma Score (GCS), shown for days 6-8 of continuous EEG (cEEG) monitoring. (b) A 57-year old woman admitted for acute subarachnoid hemorrhage (admission Hunt-Hess grade 4) from a right posterior communicating aneurysm. Admission angiography did not show vasospasm. The aneurysm was clipped on SAH day 2. No infarcts were seen on postoperative computed tomography (CT) scan. Postoperatively she had a GCS of 14. CEEG monitoring was performed from SAH days 3 to 8 . The ADR progressively decreased after day 6, particularly in the right anterior region (blue arrow), to settle into a steady trough level later that night, reflecting loss of fast frequencies and increased slowing over the right hemisphere in the raw cEEG (c; EEG2 compared with EEG1). On SAH day 6, flow velocities in the right MCA were marginally elevated $(144 \mathrm{~cm} / \mathrm{s})$, but the patient remained clinically stable with hypertensive, hypervolemic therapy (systolic blood pressure $>180 \mathrm{mmHg}$ ). On day 7, the GCS dropped from 14 to 12 and a CT scan showed a right internal capsule and hypothalamic infarction (b, Day 7). Angiography demonstrated severe distal right MCA and left vertebral artery spasm; however, due to the marked tortuosity of the parent vessels and the location of vasospasm, a decision was made not to perform angioplasty, but to infuse verapamil and papaverine. This resulted in a marked, but transient increase of the right anterior and posterior alpha/delta ratios (blue shaded area). Later that day the patient further deteriorated clinically to a GCS of 7, with a new onset left hemiparesis, and died on SAH day 9 from widespread infarction due to vasospasm. From [9] with permission.

For region specific parameters, only the relative delta percentage and the alpha/delta ratio correlated with delayed cerebral ischemia and only in the anterior derivations; however, only one patient had a posterior circulation event. When six minutes of EEG were used, a 10\% change in the alpha/delta ratio was $100 \%$ sensitive and
$76 \%$ specific for delayed cerebral ischemia. When just one minute was used, a $50 \%$ or more change from baseline yielded a sensitivity of $89 \%$ and specificity of $84 \%$. Overall, relative rather than absolute parameters appeared to be more sensitive to changes. Despite indications that hemispheric indices are predictive of clinical 
events in acute ischemic stroke, coherence performed only modestly well in this study, perhaps because of the background changes associated with $\mathrm{SAH}$ in general. Similarly, although spectral edge frequencies correlate with ischemia in acute ischemic stroke [28] and carotid endarterectomy [29] and mean frequency changes correlate with improvements in CBF [20], frequency measures were not predictive of delayed cerebral ischemia in poorgrade SAH, potentially because of lower overall frequencies at baseline. These parameters have not been adequately studied in better grade SAH, and may still hold some promise.

A recent study used a standard electrode array, excluding the posterior derivations to avoid the impact of the frequent (albeit normal) asymmetry of the posterior dominant rhythm on qEEG values [10]. Twelve patients with modified Fisher grades 3-4, corresponding to highest risk for vasospasm [43], were evaluated using the total power of the alpha frequency band (defined as $8-15 \mathrm{~Hz}$ ). Each 30 seconds, an alpha frequency value was calculated and averaged over 30 minutes. The standard deviation and the mean alpha power were then multiplied to create a 'composite alpha index'. Overall, 8 of 12 patients developed delayed cerebral ischemia. Three patients with delayed cerebral ischemia developed worsening trends in the composite alpha index more than 24 hours prior to neurodeterioration. Predictions of neurodeterioration made exclusively on clinical data were $40 \%$ sensitive, whereas the addition of qEEG data increased this to $67 \%$ with a specificity of $73 \%$.

\section{Future directions}

Both the relative alpha variability and the composite alpha indices were derived in an effort to generate a discrete trending value accessible to other members of the patient care team. Unfortunately, both measures require artifact detection based on visual inspection by electroencephalographers. While post-stimulation clips can be easily evaluated for artifact at the time of the stimulation, this may be labor intensive and does not provide a continuous measure. Future studies of ischemia detection should focus on discrete and easily interpretable values generated in real-time, accessible to the neurophysiologist, intensivists, and the nursing staff.

Based on qEEG data in ischemia, it appears that regional power values may be more specific to the nature and size of the underlying lesion and, therefore, correlate with later functional outcome; however, they may not be sensitive enough to use alone in ischemia detection [9]. Hemispheric power differences highlight acute ischemic changes, but correlate less well with the subtle, multifocal, and more gradual changes seen in SAH. qEEG frequency values (such as spectral edge frequencies) may be useful to detect changes in relatively healthy brain
[29], but it is not clear that these are helpful in more widespread pathology such as SAH. Some values (such as global increases in theta range frequency) may have a relationship to the penumbra and, therefore, be useful in the pre-infarct stage of ischemia in good-grade SAH [36] but this has not been adequately studied. To develop a rational approach to the detection of ischemia, a combination of regional, hemispheric, and total measures of both power and frequency may be needed.

An attempt should be made to integrate qEEG parameters into the multimodal monitoring infrastructure available in some ICUs. Electrophysiological changes should be interpreted in the context of blood pressure, systemic oxygenation, intracranial or cerebral perfusion pressures, cerebral measures of oxygen tension, or microdialysis. More recently, intra-cortical electroencephalography has provided valuable information on both seizure and ischemia detection: In a series of 5 patients who had intra-cortical electroencephalography, a change in the alpha/delta ratio of $25 \%$ sustained over 4 hours was the most accurate parameter in detecting vasospasm between 1 and 3 days prior to confirmation with angiography [48]. Subdural electrodes have detected periinfarct depolarizations and cortical spreading depression [16], phenomena invisible to scalp EEG but that might play a role in delayed cerebral ischemia after SAH. Interestingly, using subdural electrodes these researchers were also able to detect an abnormal hemodynamic response when analyzing the cortical spreading depression signal in relation to ischemia from vasospasm [16].

Ultimately, ischemia detection requires real-world validation. Software currently exists that allows real-time quantification of qEEG parameters. The numerical values generated by qEEG can be used to set threshold alarms such that when a specified value is reached for more than a specified period of time (e.g., to avoid confusion with artifact), an alert is sent to a treating member of the ICU team. This should be done in the context of a prospective and randomized trial that compares integrating qEEG into a novel algorithm versus best clinical practice, for example comparing different triggers to obtain established confirmatory assessments of vasospasm-associated delayed cerebral ischemia, such as CT angiography, CT perfusion, or conventional angiography.

\section{Conclusion}

EEG is a very promising tool for monitoring brain function in real-time in the ICU. There are characteristic changes that occur on EEG in response to brain ischemia, correlating with $\mathrm{CBF}$ and brain metabolism. Although raw EEG evaluations are time consuming, quantification of EEG features in real-time has been incorporated into many of the standard EEG software packages. Some of these quantitative parameters have also been found to 
correspond to physiologic changes associated with ischemia. Sensitive techniques are needed to detect cerebral ischemia, for example, in vasospasm-associated delayed cerebral ischemia after SAH. Early evidence suggests that qEEG may be sensitive enough to allow pre-clinical detection of delayed cerebral ischemia from vasospasm. This approach may be utilized to widen the window of opportunity in order to prevent permanent neuronal damage in a variety of clinic scenarios.

\section{Abbreviations}

ADR, alpha/delta ratio; ATP, adenosine triphosphate; BSI, brain symmetry index; CBF, cerebral blood flow; $C E E G$, continuous EEG monitoring; $\mathrm{CMRO}_{2}$, cerebral rate of oxygen metabolism; CPP, cerebral perfusion pressure; $C T$, computed tomography; DCl, delayed cerebral ischemia; DWI MRI, diffusionweighted magnetic resonance images; EEG, electroencephalography; GCS, Glasgow Coma Score; HH, Hunt-Hess Grade; ICU, intensive care unit; MAP mean arterial pressure; MCA, middle cerebral artery; mF, Modified Fisher Grade; NIHSS, National Institutes of Health Stroke Scale; PET, positron emission tomography; qEEG, quantitative EEG; RAWOD, regional attenuation of faster frequencies without delta; $\mathrm{SAH}$, subarachnoid hemorrhage; TCD, transcranial Doppler ultrasonography; tPA, plasminogen activator; VAP, ventilator-acquired pneumonia.

\section{Competing interests}

The authors declare there are no competing interests.

\section{Author details}

'Columbia University College of Physicians \& Surgeons, Division of Critical Care Neurology, New York, NY, USA. ²Department of Neurology, Columbia University, New York, NY, USA.

Published: 20 March 2012

\section{References}

1. Friedman D, Claassen J, Hirsch LJ: Continuous electroencephalogram monitoring in the intensive care unit. Anesth Analg 2009, 109:506-523.

2. Hossmann KA: Viability thresholds and the penumbra of focal ischemia. Ann Neurol 1994, 36:557-565

3. Sharbrough FW, Messick JM Jr, Sundt TM Jr: Correlation of continuous electroencephalograms with cerebral blood flow measurements during carotid endarterectomy. Stroke 1973, 4:674-683.

4. Jordan KG: Emergency EEG and continuous EEG monitoring in acute ischemic stroke. J Clin Neurophysiol 2004, 21:341-352.

5. Frontera JA, Fernandez A, Schmidt JM, et al.: Defining vasospasm after subarachnoid hemorrhage: what is the most clinically relevant definition? Stroke 2009, 40:1963-1968.

6. Schmidt JM, Wartenberg KE, Fernandez A, et al:: Frequency and clinical impact of asymptomatic cerebral infarction due to vasospasm after subarachnoid hemorrhage. J Neurosurg 2008, 109:1052-1059.

7. Labar DR, Fisch BJ, Pedley TA, Fink ME, Solomon RA: Quantitative EEG monitoring for patients with subarachnoid hemorrhage. Electroencephalogr Clin Neurophysiol 1991, 78:325-332.

8. Vespa PM, Nuwer MR, Juhasz C, et al:: Early detection of vasospasm after acute subarachnoid hemorrhage using continuous EEG ICU monitoring. Electroencephalogr Clin Neurophysiol 1997, 103:607-615.

9. Claassen J, Hirsch LJ, Kreiter KT, et al:: Quantitative continuous EEG for detecting delayed cerebral ischemia in patients with poor-grade subarachnoid hemorrhage. Clin Neurophysio/ 2004, 115:2699-2710.

10. Rathakrishnan R, Gotman J, Dubeau F, Angle M: Using continuous electroencephalography in the management of delayed cerebral ischemia following subarachnoid hemorrhage. Neurocrit Care 2011, 14:152-161.

11. Amzica F, Lopes da Silva FH: Cellular substrates of brain rhythms. In Niedermeyer's Electroencephalography: Basic Principles, Clinical Applications, and Related Fields. 6th edition. Edited by Niedermeyer E, Schomer DL, Lopes da Silva FH. Philadelphia: Wolters Kluwer/Lippincott Williams \& Wilkins Health; 2010:33-64.

12. Evans BM: Patterns of arousal in comatose patients. J Neurol Neurosurg Psychiatry 1976, 39:392-402.
13. Schneider AL, Jordan KG: Regional attenuation without delta (RAWOD): a distinctive EEG pattern that can aid in the diagnosis and management of severe acute ischemic stroke. Am J Electroneurodiagnostic Technol 2005, 45:102-117.

14. Nagata K, Tagawa K, Hiroi S, Shishido F, Uemura K: Electroencephalographic correlates of blood flow and oxygen metabolism provided by positron emission tomography in patients with cerebral infarction. Electroencephalogr Clin Neurophysiol 1989, 72:16-30.

15. Powers WJ: Cerebral hemodynamics in ischemic cerebrovascular disease. Ann Neurol 1991, 29:231-240.

16. Dreier JP, Major S, Manning A, et al.: Cortical spreading ischaemia is a novel process involved in ischaemic damage in patients with aneurysmal subarachnoid haemorrhage. Brain 2009, 132:1866-1881.

17. Kohno K, Hoehn-Berlage M, Mies G, BackT, Hossmann KA: Relationship between diffusion-weighted MR images, cerebral blood flow, and energy state in experimental brain infarction. Magn Reson Imaging 1995, 13:73-80.

18. Finnigan SP, Rose SE, Chalk JB: Rapid EEG changes indicate reperfusion after tissue plasminogen activator injection in acute ischaemic stroke. Clin Neurophysiol 2006, 117:2338-2339.

19. de Vos CC, van Maarseveen SM, Brouwers PJ, van Putten MJ: Continuous EEG monitoring during thrombolysis in acute hemispheric stroke patients using the brain symmetry index. J Clin Neurophysiol 2008, 25:77-82.

20. Wood JH, Polyzoidis KS, Epstein CM, Gibby GL, Tindall GT: Quantitative EEG alterations after isovolemic-hemodilutional augmentation of cerebral perfusion in stroke patients. Neurology 1984, 34:764-768.

21. Bricolo A, Turazzi S, Faccioli F: Combined clinical and EEG examinations for assessment of severity of acute head injuries. Acta Neurochir Suppl (Wien) 1979, 28:35-39.

22. Bergamasco B, Bergamini L, Doriguzzi T, Sacerdote I: The sleep cycle in coma: prognostic value. Electroencephalogr Clin Neurophysiol 1968, 25:87.

23. Burghaus L, Hilker R, Dohmen C, et al.: Early electroencephalography in acute ischemic stroke: prediction of a malignant course? Clin Neurol Neurosurg 2007, 109:45-49.

24. Cillessen JP, van Huffelen AC, Kappelle $\amalg$, Algra A, van Gijn J: Electroencephalography improves the prediction of functional outcome in the acute stage of cerebral ischemia. Stroke 1994, 25:1968-1972.

25. Pohlmann-Eden B, Hoch DB, Cochius Jl, Chiappa KH: Periodic lateralized epileptiform discharges-a critical review. J Clin Neurophysiol 1996, 13:519-530.

26. Scheuer ML, Wilson SB: Data analysis for continuous EEG monitoring in the ICU: seeing the forest and the trees. J Clin Neurophysiol 2004, 21:353-378.

27. Lopes da Silva FH: EEG analysis: theory and practice. In Niedermeyer's Electroencephalography: Basic Principles, Clinical Applications, and Related Fields. 6th edition. Edited by Niedermeyer E, Schomer DL, Lopes da Silva FH. Philadelphia: Wolters Kluwer/Lippincott Williams \& Wilkins Health; 2010:1147-1178

28. Tolonen U, Sulg IA: Comparison of quantitative EEG parameters from four different analysis techniques in evaluation of relationships between EEG and CBF in brain infarction. Electroencephalogr Clin Neurophysiol 1981, 51:177-185

29. Laman DM, Wieneke GH, van Duijn H, Veldhuizen RJ, van Huffelen AC: QEEG changes during carotid clamping in carotid endarterectomy: spectral edge frequency parameters and relative band power parameters. J Clin Neurophysiol 2005, 22:244-252.

30. Diedler J, Sykora M, Bast T, et al:: Quantitative EEG correlates of low cerebral perfusion in severe stroke. Neurocrit Care 2009, 11:210-216.

31. van Putten MJ, Tavy DL: Continuous quantitative EEG monitoring in hemispheric stroke patients using the brain symmetry index. Stroke 2004, 35:2489-2492

32. Sheorajpanday RV, Nagels G, Weeren AJ, van Putten MJ, De Deyn PP Reproducibility and clinical relevance of quantitative EEG parameters in cerebral ischemia: a basic approach. Clin Neurophysio/ 2009, 120:845-855.

33. Sheorajpanday RV, Nagels G, Weeren AJ, De Deyn PP: Quantitative EEG in ischemic stroke: correlation with infarct volume and functional status in posterior circulation and lacunar syndromes. Clin Neurophysio/ 2011 122:884-890.

34. Finnigan SP, Walsh M, Rose SE, Chalk JB: Quantitative EEG indices of subacute ischaemic stroke correlate with clinical outcomes. Clin Neurophysiol 2007, 118:2525-2532.

35. Finnigan SP, Rose SE, Walsh M, et al:: Correlation of quantitative EEG in acute ischemic stroke with 30-day NIHSS score: comparison with diffusion and 
perfusion MRI. Stroke 2004, 35:899-903.

36. Sheorajpanday RV, Nagels G, Weeren AJ, De Surgeloose D, De Deyn PP: Additional value of quantitative EEG in acute anterior circulation syndrome of presumed ischemic origin. Clin Neurophysiol 2010 121:1719-1725.

37. Nuwer MR, Jordan SE, Ahn SS: Evaluation of stroke using EEG frequency analysis and topographic mapping. Neurology 1987, 37:1153-1159.

38. Murri L, Gori S, Massetani R, Bonanni E, Marcella F, Milani S: Evaluation of acute ischemic stroke using quantitative EEG: a comparison with conventional EEG and CT scan. Neurophysiol Clin 1998, 28:249-257.

39. Sheorajpanday RV, Nagels G, Weeren AJ, van Putten MJ, De Deyn PP: Quantitative EEG in ischemic stroke: correlation with functional status after 6 months. Clin Neurophysiol 2011, 122:874-883.

40. Oddo M, Carrera E, Claassen J, Mayer SA, Hirsch LJ: Continuous electroencephalography in the medical intensive care unit. Crit Care Med 2009, 37:2051-2056.

41. Taccone FS, Su F, Pierrakos C, et al:: Cerebral microcirculation is impaired during sepsis: an experimental study. Crit Care 2010, 14:R140

42. Washington CW, Zipfel GJ: Detection and monitoring of vasospasm and delayed cerebral ischemia: a review and assessment of the literature. Neurocrit Care 2011, 15:312-317.

43. Claassen J, Bernardini GL, Kreiter K, et al:: Effect of cisternal and ventricular blood on risk of delayed cerebral ischemia after subarachnoid hemorrhage: the Fisher scale revisited. Stroke 2011, 32:2012-2020.
44. Carrera E, Schmidt JM, Oddo M, et al:: Transcranial Doppler for predicting delayed cerebral ischemia after subarachnoid hemorrhage. Neurosurgery 2009, 65:316-323.

45. Claassen J, Hirsch $\sqcup$, Frontera JA, et al:: Prognostic significance of continuous EEG monitoring in patients with poor-grade subarachnoid hemorrhage. Neurocrit Care 2006, 4:103-112.

46. Tettenborn B, Niedermeyer E, Schomer DL: EEG analysis: theory and practice. In Niedermeyer's Electroencephalography: Basic Principles, Clinical Applications, and Related Fields. 6th edition. Edited by Niedermeyer E, Schomer DL, Lopes da Silva FH. Philadelphia: Wolters Kluwer/Lippincott Williams \& Wilkins Health; 2011:351-374.

47. Rivierez M, Landau-Ferey J, Grob R, Grosskopf D, Philippon J: Value of electroencephalogram in prediction and diagnosis of vasospasm after intracranial aneurysm rupture. Acta Neurochir (Wien) 1991, 110:17-23.

48. Stuart RM, Waziri A, Weintraub D, et al: Intracortical EEG for the detection of vasospasm in patients with poor-grade subarachnoid hemorrhage. Neurocrit Care 2010, 13:355-358

doi:10.1186/cc11230

Cite this article as: Foreman B, Claassen J: Quantitative EEG for the detection of brain ischemia. Critical Care 2012, 16:216 